\title{
Vibronic state assisted resonant transport in molecules strongly anchored at an electrode
}

\author{
Shiyong Wang, ${ }^{1}$ Weihua Wang, ${ }^{1}$ Yuning Hong, ${ }^{2}$ Ben Zhong Tang, ${ }^{2}$ and Nian Lin ${ }^{1, *}$ \\ ${ }^{1}$ Department of Physics, The Hong Kong University of Science and Technology, Hong Kong, China \\ ${ }^{2}$ Department of Chemistry, The Hong Kong University of Science and Technology, Hong Kong, China \\ (Received 27 October 2010; revised manuscript received 8 February 2011; published 15 March 2011)
}

\begin{abstract}
We present a single-molecule study demonstrating that resonant charge transport through vibronic states takes place in molecules that are strongly anchored at an electrode. The molecules are adsorbed on a $\mathrm{CuN} / \mathrm{Cu}(100)$ surface, with the molecular terminal group attaching to the $\mathrm{Cu}$ ridge. We use a scanning tunneling microscope to inject charges into the highest occupied molecular orbital and excite vibronic transitions. The charges are then transferred in a single-electron process through either a single or a double intramolecular channel, depending on molecular adsorption configuration. These phenomena are explained in terms of localized molecular orbitals and an effective intramolecular barrier.
\end{abstract}

DOI: 10.1103/PhysRevB.83.115431

PACS number(s): 85.65.+h, 68.37.Ef, 73.23.Hk, 73.63.Rt

\section{INTRODUCTION}

Charge transport in single molecules has been intensively studied in the past few decades from the perspective of single molecules being used as electronic devices. ${ }^{1-5}$ Recently, electron-phonon or electron-vibration interactions in molecular charge transport have attracted increasing attention, since this effect involves rich phenomena, including inelastic tunneling, charging, vibronic transition, switching, and negative differential resistance. ${ }^{6-18}$ In a metal-molecule-metal junction, the strength of molecule-metal coupling plays a decisive role in charge transport as well as electron-phonon interactions. ${ }^{2-5}$ In general, when this coupling is relatively weak the individual vibronic levels can be resolved as they align with the metal Fermi level, whereas for strong coupling the vibronic levels cannot be resolved, due to severe level broadening. ${ }^{19,20}$ This understanding needs to be examined carefully for multicomponent molecules: Molecules used in single-molecule devices usually consist of multiple chemical groups, which often provide localized molecular orbitals (MOs). The MO localization may result in an intramolecular barrier, which can effectively enhance resonant transport and/or electron-phonon interactions. For example, it was predicted that the vibronic signatures become visible in a molecule that is strongly coupled to gold electrodes via alkanethiol bridges. ${ }^{21}$ Experimentally, it was demonstrated that in a break-junction configuration, charge transport of strongly coupled molecules that contain spacer groups exhibit Coulomb blockade behavior or vibronic characteristics. ${ }^{22,23}$ However, the molecule-electrode contact could not be characterized directly in these studies.

In this paper, we demonstrate that vibronic state mediated resonant transport takes place in multicomponent molecules that are strongly adsorbed on a metal surface. The characteristics of the molecule-metal contacts were revealed directly using a cryogenic scanning tunneling microscope (STM). The molecule used in this study is bis-terpyridine tetra-phenyl ethylene (BTP-TPE). As shown in Fig. 1(a), this molecule consists of five molecular groups: two terpyridine (tp) terminal groups linked to a central tetra-phenyl ethylene (tpe) group through two phenyl (ph) spacer groups. As deposited on a surface of $\mathrm{CuN} / \mathrm{Cu}(100)$, some molecules were strongly coupled to the $\mathrm{Cu}$ regions via either single- or double-reactive tp terminal group(s), while the central tpe group was weakly adsorbed on the $\mathrm{CuN}$ region. In such a configuration, the tpe group could be charged and excited to vibronic states by the STM tip at resonant conditions. This phenomenon is attributed to the localized MOs and an effective intramolecular barrier. As illustrated in Fig. 1(b), due to the MO localization and the effective intramolecular barrier, a MO remains as a sharp state as the molecular terminal group is coupled strongly to the electrode. This level thus offers a channel for the resonant transport, which is associated with charging and vibronic excitation. Furthermore, the conductance as well as vibronic signatures of the doubly contacted BTP-TPE molecules is found to be substantially enhanced compared to those of the singly contacted ones.

\section{EXPERIMENT}

The experiments were carried out in an ultrahigh vacuum STM system (Omicron) operated at $4.9 \mathrm{~K}$. A single-crystal $\mathrm{Cu}(100)$ sample was used as a substrate for the $\mathrm{CuN}$ growth. The copper surface was cleaned by cycles of $\mathrm{Ar}^{+}$sputtering and annealing to $\sim 800 \mathrm{~K}$. CuN nanoislands were grown on $\mathrm{Cu}(100)$ following the reported recipe ${ }^{24}$ The molecules were thermally evaporated onto the $\mathrm{CuN} / \mathrm{Cu}(100)$ substrate held at $\sim 30$ or $\sim 100 \mathrm{~K}$. The evaporation temperature for BTPTPE molecules is $700 \mathrm{~K}$. Samples were prepared with low molecule coverage, so that it was easy to find single molecules on $\mathrm{CuN}$ islands or copper trenches. The STM topographic data were acquired in constant current mode. The $d I / d V$ data were measured using a lock-in amplifier with a sine modulation of $1.5 \mathrm{kHz}$ at $5 \mathrm{mV}$.

\section{RESULTS AND DISSCUSSIONS}

\section{A. Adsorption configurations}

As shown in Fig. 2, the $\mathrm{CuN}$ nanoislands are of $\sim 5 \mathrm{~nm}$ in size and separated by $\mathrm{Cu}$ ridges of $\sim 1 \mathrm{~nm}$ in width. Although they form only a monatomic-thick layer, the $\mathrm{CuN}$ nanoislands behave as insulators. ${ }^{25}$ STM topographs reveal that tp groups adsorb on the $\mathrm{Cu}$ ridges more favorably. This is because the terpyridine group tends to form coordination bonds with metal. ${ }^{26}$ In general, four distinctive adsorption configurations 
(a)

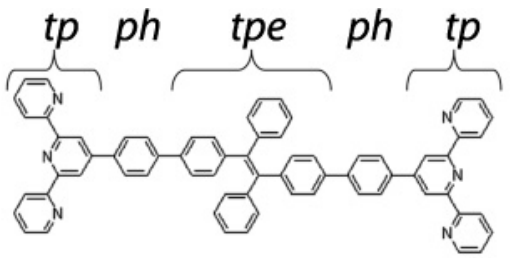

(b) multi-component
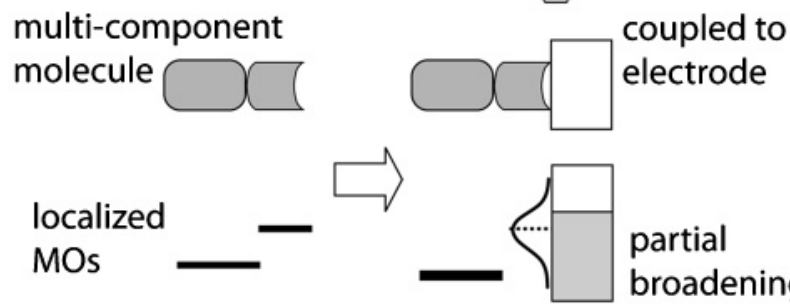

partial broadening

FIG. 1. (a) Molecular structure of BTP-TPE. (b) As a multicomponent molecule with localized MOs is coupled to an electrode via its terminal group, the strong coupling broadens the MO localized at the terminal group, but an effective intramolecular barrier retains as the sharp state of the other MO.

are observed, as shown in Figs. 2(a)-2(d): M1 is a molecule fully adsorbed on a $\mathrm{Cu}$ ridge; $\mathrm{M} 2$ is a molecule fully adsorbed on a $\mathrm{CuN}$ nanoisland; $\mathrm{M} 3$ is a molecule with one tp group adsorbed on a $\mathrm{Cu}$ ridge and the rest of its parts on a $\mathrm{CuN}$ nanoisland; and M4 is a molecule with both tp groups on $\mathrm{Cu}$ ridges and the tpe group on a $\mathrm{CuN}$ nanoisland. These four configurations give rise to four types of molecule-Cu contacts, as illustrated by the models in Figs. 2(e)-2(h).

Figures 2(e)-2(h) show the site-dependent tunneling spectra acquired with the tip positioned above the central tpe group (red curve) or above the two terminal tp groups (black and green curves) of each type of molecule. All three spectra of the M1 molecule are featureless, aside from the uprising at higher bias voltages (note that the $\mathrm{CuN}$ shows a strong peak at $2.2 \mathrm{~V}$ ), meaning the molecule-Cu coupling is strong enough to quench the intrinsic molecular states. In sharp contrast, the spectra of the M2 molecule exhibit a $-1.8 \mathrm{~V}$ peak at tpe and a $1.6-\mathrm{V}$ peak at both tp groups. Previously, we reported that the highest occupied MO (HOMO) of BTP-TPE is at $-1.8 \mathrm{~V}$ and localized at tpe, and an unoccupied state is at $1.3 \mathrm{~V}$ and localized at tp. ${ }^{27}$ The tunneling spectra of M2 indicate that the intrinsic molecular states are only slightly altered as the molecule becomes adsorbed on the $\mathrm{CuN}$ nanoisland, which infers a rather weak molecule-CuN interaction. It is worthwhile to point out that the conductance peaks are accompanied by series satellite features at higher energies. This behavior reflects vibronic characteristics that will be discussed in detail later.

Interestingly, the tunneling spectra acquired at the tpe group of the M3 or M4 molecule reproduce the intrinsic features of tpe independent of the adsorption of the tp groups. For example, one of the tp groups of the M3 molecule is adsorbed on the $\mathrm{Cu}$ ridge and shows a featureless spectrum, indicating a strong $\mathrm{Cu}$-tp coupling, whereas the tpe group, which is adsorbed on the $\mathrm{CuN}$, shows a pronounced $-1.8 \mathrm{~V}$ peak. So, the strong Cu-tp coupling only suppresses the state localized at this tp group but does not perturb the HOMO state, which is localized at tpe; i.e., the two states are virtually decoupled. In addition, both M3 and M4 exhibit satellite peaks alongside the main $-1.8 \mathrm{~V}$ peak, indicating vibronic excitations take place in M3 or M4, too. This observation implies that the vibronic states have a considerable lifetime, which is associated with a sharp energy level, despite the strong coupling of the terminal tp group (s) to the $\mathrm{Cu}$ electrode. We interpret this behavior in terms of the localization of the MOs and the effective intramolecular barrier between the groups of tp and tpe. These two factors allow the HOMO, which is localized at tpe, to remain as a rather sharp state.

\section{B. Single-electron tunneling}

In the M2 configuration, the molecule is sandwiched between a vacuum gap and the $\mathrm{CuN}$ layer as the STM tip is placed over the molecule. So, the molecule is in a double-barrier-tunneling (DBT) junction, as shown by the inset model in Fig. 3(a). In the presence of insulating $\mathrm{CuN}$ film, the
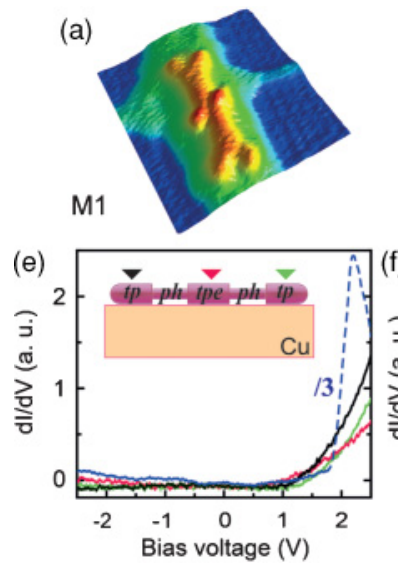
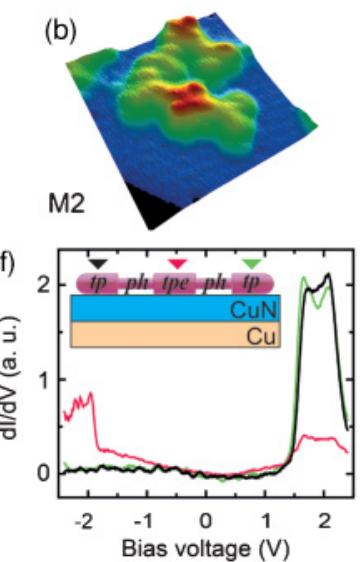

(c)
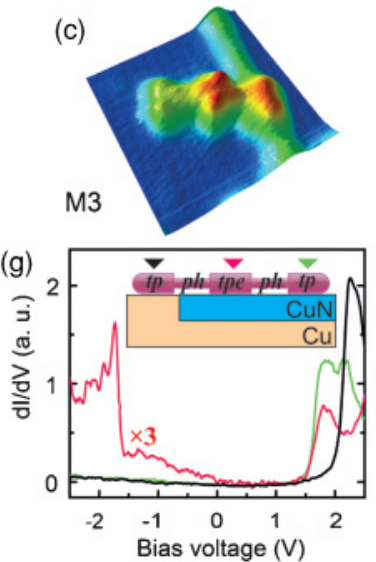
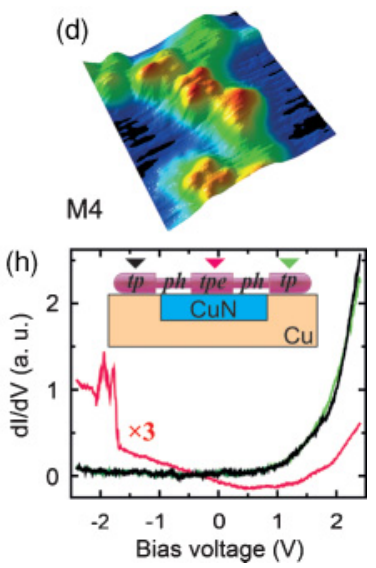

FIG. 2. (Color online) (a)-(d) STM topographic images showing the molecules of four adsorption types of M1, M2, M3, and M4 on the $\mathrm{CuN} / \mathrm{Cu}(100)$ substrate (the area in blue is $\mathrm{CuN}$ ). (Image size: $5.5 \times 5.5 \mathrm{~nm}^{2}$. Imaging conditions: $V_{b}=-1.0 \mathrm{~V}$ and $I=0.3 \mathrm{nA}$ for $\mathrm{M} 1$; $V_{b}=-1.0 \mathrm{~V}$ and $I=0.01 \mathrm{nA}$ for M2; $V_{b}=1.0 \mathrm{~V}$ and $I=0.01 \mathrm{nA}$ for M3; and $V_{b}=1.5 \mathrm{~V}$ and $I=0.12 \mathrm{nA}$ for M4.) (e)-(h) Site-dependent tunneling spectra acquired at the tp groups (black and green curves) and the tpe group (red curves) of the four molecules. The dashed blue curve in (e) is a spectrum measured at clean $\mathrm{CuN}$. (The spectra were taken with a lock-in amplifier with $f=1.5 \mathrm{kHz}$ and $v_{\text {mod }}=30 \mathrm{mV}$.) Inset models: contact configuration of each adsorption ( $\mathrm{CuN}$ in blue and $\mathrm{Cu}$ in orange). 

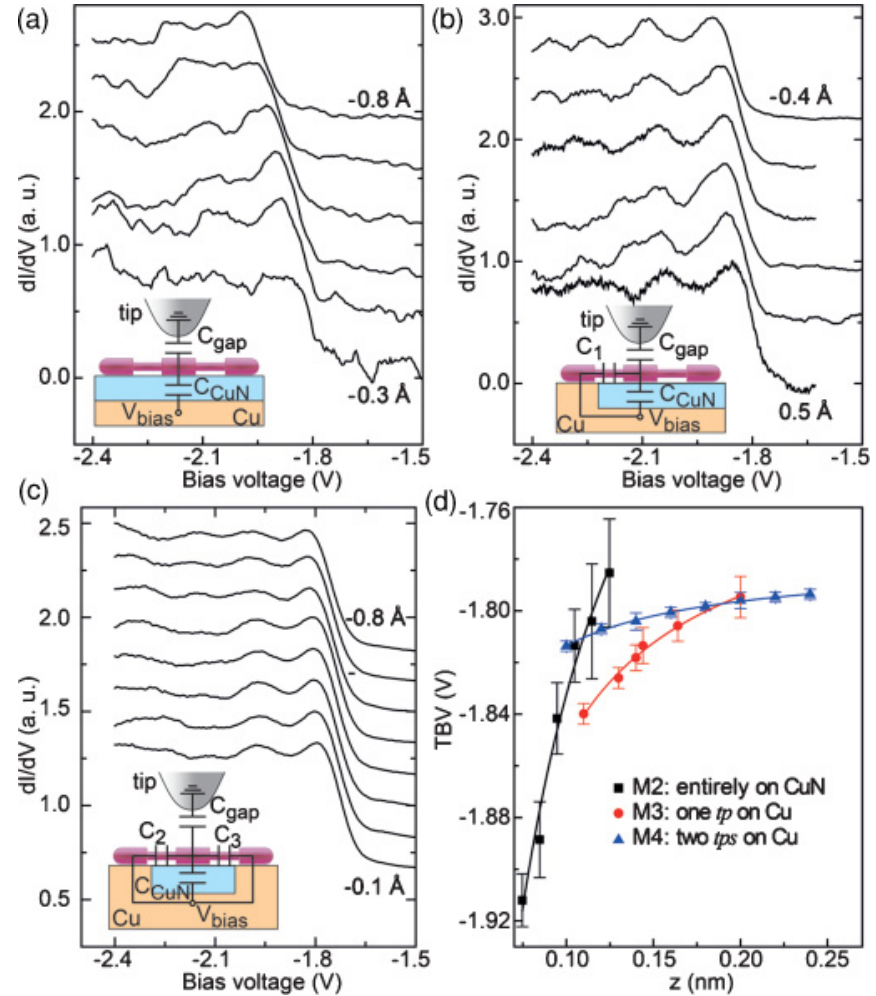

FIG. 3. (Color online) (a)-(c) $d I / d V$ spectra acquired at tpe with different tip-molecule separations of the M2, M3, and M4 molecules, respectively. The spectra are shifted vertically for clarity. Inset: the effective DBT junction and the equivalent circuit model for each molecule. (d) TBV as a function of $z$ of M2 (square), M3 (circle), and M4 (triangle). Curves: theoretical fittings given by Eqs. (1a), (2), and (3) $(\mathrm{CuN}$ in blue and $\mathrm{Cu}$ in orange).

coupling to both of the electrodes is small enough that the charge transport through a molecule at resonant conditions is a single-electron sequential tunneling process. ${ }^{9-11},{ }^{28-30}$ This single-electron transport was explained as a two-step tunneling mechanism using a parallel-plate capacitor model. ${ }^{9,28,29}$ In this model, the key factor determining threshold bias voltages (TBVs) is the initial tunneling step, which has two cases: Case I is defined as the initial tunneling step between the copper substrate and the molecule, where TBVs are given by

$$
e V_{b}=E_{0}\left(1+\frac{z C_{\mathrm{CuN}}}{\varepsilon_{0} s}\right)
$$

case II is defined as the initial tunneling step between the STM tip and the molecule, where the TBVs are given by

$$
e V_{b}=-E_{0}\left(1+\frac{\varepsilon_{0} s}{{ }_{z} C_{\mathrm{CuN}}}\right) .
$$

In both cases, $E_{0}$ is the ionization energy, $z$ is the tip-molecule separation, $s$ is the cross-sectional area of the tpe group $\left(0.8 \mathrm{~nm}^{2}\right), \varepsilon_{0}$ is the dielectric constant of vacuum, and $C_{\mathrm{CuN}}$ is the capacitance of the insulating $\mathrm{CuN}$ film. Based on Eqs. (1a) and (1b), it is obvious that the TBVs are sensitive to $z$ offsets for both cases: The TBVs are proportional to $z$ offsets for case I conduction and inversely proportional to $z$ offsets for case II conduction. As shown in Fig. 3(d), an inverse proportional relation was observed between TBVs and $z$ offsets for M2, which indicates that the initial tunneling step is between the STM tip and molecule. Figure 3(a) shows a series of $d I / d V$ spectra acquired at the tpe group with a different tip-molecule separation $z$, revealing that the TBV of the resonant tunneling shifts toward a more negative value at a narrower tip-molecule separation, which further confirms the initial tunneling step belonged to case II conduction.

For the molecules M3 and M4, although the terminal molecular group(s) is (are) coupled strongly with $\mathrm{Cu}$, the effective intramolecular barrier, together with the $\mathrm{CuN}$ layer, serves as the second barrier. Therefore, M3 or M4 is virtually in a DBT junction, too. We define an intramolecular capacitance to model the DBT junction of M3 and M4, as illustrated in the inset models in Figs. 3(b) and 3(c). By taking into account the intramolecular capacitance as shown in these models, the TBVs of M3 and M4 can be derived respectively as

$$
e V_{b}=-E_{0}\left[1+\frac{\varepsilon_{0} s}{z\left(C_{\mathrm{CuN}}+C_{1}\right)}\right]
$$

and

$$
e V_{b}=-E_{0}\left[1+\frac{\varepsilon_{0} s}{z\left(C_{\mathrm{CuN}}+C_{1}+C_{2}\right)}\right]
$$

Figure 3(d) shows the shifting of the TBVs of M2 (square), M3 (dot), and M4 (triangle) as a function of tip-molecule separation $z$. One can see the shifting rate of M2 is largest, followed by M3 and then M4. This trend can be understood by the increasing capacitance in the denominator in Eqs. (1b), (2), and (3).

\section{Vibronic state mediated resonant transport}

From the above discussion, the inversely proportional relation between TBVs and $z$ offsets confirms that the first tunneling step is between the STM tip and the molecule. For conduction through tpe, at $V_{b}<0$, once the TBV is reached, a HOMO electron will first tunnel through the vacuum barrier from molecular HOMO into the STM tip. Due to the presence of the second barrier, the tpe group can retain the charged state until the second tunneling step takes place, in which an electron tunnels from the $\mathrm{Cu}$ substrate through the $\mathrm{CuN}$ layer. As the bias voltage continues increasing beyond the resonance until reaching a molecular vibrational level, a new tunneling channel is opened up. ${ }^{9-11}$ This results in vibronic peaks in the differential tunneling spectra below $-1.8 \mathrm{~V}$.

Detailed inspection of Figs. 3(a)-3(c) reveals that the satellite features appear as a set of equally spaced peaks. These conductance peaks correspond to different vibrational levels. Varying the tip-molecule separation, we found that the distances between two neighbor satellite peaks are slightly different, which is due to voltage division in the DBT junction. Taking into account the voltage division, the peak spacing amounts to $191 \pm 6 \mathrm{meV}$ for all adsorption configurations. This value is very close to the tpe group's $\mathrm{C}=\mathrm{C}$ bond-stretching mode of $200 \mathrm{meV}$.

\section{Intramolecular capacitance and conductance}

Fitting the data of M2 (square) using Eq. (1b), we obtained $E_{0}=-1.6 \mathrm{~V}$ and $C_{\mathrm{CuN}}=0.5 \mathrm{aF}$. Taking the thickness of the $\mathrm{CuN}$ layer as $0.18 \mathrm{~nm},{ }^{25}$ we estimate the dielectric 


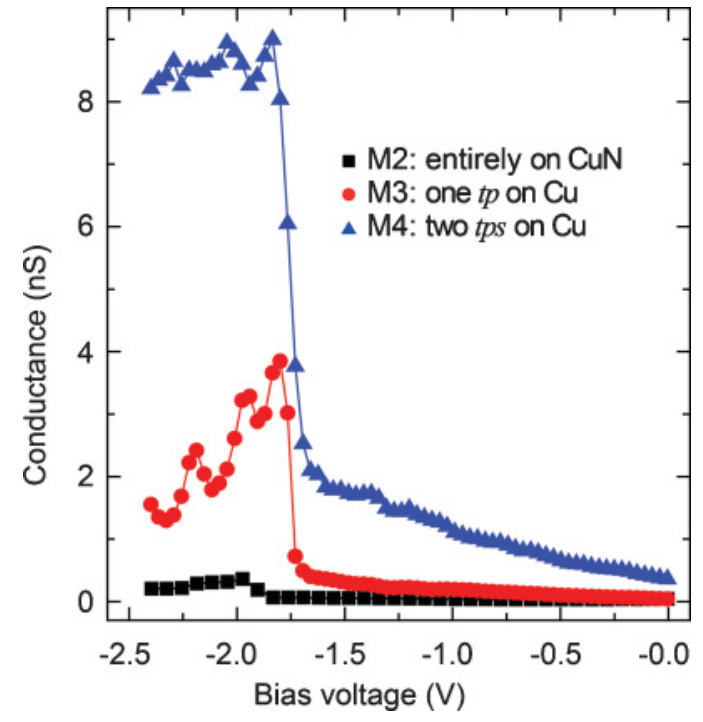

FIG. 4. (Color online) Differential conductance calculated from $I-V$ spectra of M2, M3, and M4 acquired at a constant tip-molecule separation $z=0.10 \mathrm{~nm}$.

constant of $\mathrm{CuN}$ as 11. This value is in good agreement with the reported dielectric constant of nitride compounds, e.g., 9.14 for aluminum nitride. ${ }^{31}$ Fitting the data of M3 and M4 using Eqs. (2) and (3), respectively, we obtain $E_{0}=-1.7 \mathrm{~V}$ and $C_{\mathrm{CuN}}+C_{1}=1.4 \mathrm{aF}$ for $\mathrm{M} 3$, and $E_{0}=$ -1.8 Vand $C_{\mathrm{CuN}}+C_{2}+C_{3}=3.4 \mathrm{aF}$ for M4. Deducing $C_{\mathrm{CuN}}$ $(0.5 \mathrm{aF})$, we derive $C_{1}=0.9 \mathrm{aF}$ in $\mathrm{M} 3$ and $C_{2}+C_{3}=3.4 \mathrm{aF}$ in M4. The difference in effective capacitance of M3 and M4 manifests the different charge transport channel in these two configurations: In the second tunneling step, in M3 the electron hops through a singly contacted molecular channel, whereas in M4 the electron hops through a doubly contacted molecular channel. In total, eight M3 and nine M4 molecules were examined. The capacitance varied from 0.9 to $1.4 \mathrm{aF}$ for the M3-type molecules and from 1.8 to $3.4 \mathrm{aF}$ for the M4-type molecules. The large variation may be caused by the different contact configurations. Nevertheless, the obtained values are in fair agreement with previously reported molecular capacitance. $^{32}$

Next, we evaluate the conductance of M2, M3, and M4. The conductance can be expressed as $\frac{2 e^{2}}{h} T$, where $T$ denotes the transmission of the conductance junction. Figure 4 shows the typical differential conductance calculated from $I-V$ spectra that were acquired at a same tip-molecule separation of $0.10 \mathrm{~nm}$ for the three types of molecules. Since the vacuum gap transmission is a constant in the three cases, the conductance can be used to quantitatively compare the transmission from the tpe to the $\mathrm{Cu}$ substrate in the three configurations. It can be seen that the conductance of M2 is very small $(<0.5 \mathrm{nS})$, which reflects the limited tunneling through the $\mathrm{CuN}$ layer. M4 (M3) shows a conductance of $9 \mathrm{nS}(4 \mathrm{nS})$ at the resonance, so the transmission is significantly enhanced by the tp-Cu contact(s). This enhancement also results in more pronounced vibronic signatures. Furthermore, the larger conductance of M4 indicates that the doubly coupled molecule is more conductive than the singly coupled one.

\section{CONCLUSION}

In summary, our results reveal that single-electron resonant charge transport involving electron-phonon interactions takes place in multicomponent molecules that are strongly anchored at a metal surface. This phenomenon is attributed to localized MOs and intramolecular barriers, which effectively preserve a sharp HOMO state even as the molecular terminal group(s) is (are) strongly anchored at the electrode. This finding highlights the relevance of the MO's spatial characteristics in molecular conductance and calls for a careful description of moleculeelectrode coupling.

\section{ACKNOWLEDGMENT}

This work was supported financially by the RGC of Hong Kong (Grant No. 602008). *phnlin@ust.hk

${ }^{1}$ A. Aviram and M. A. Ratner, Chem. Phys. Lett. 29, 277 (1974).

${ }^{2}$ E. A. Weiss, M. R. Wasielewski, and M. A. Ratner, Top. Curr. Chem. 257, 103 (2005).

${ }^{3}$ A. Aviram and M. Ratner, Molecular Electronics: Science and Technology (New York Academy of Sciences, New York, 1998).

${ }^{4}$ N. J. Tao, Nat. Nanotechnol. 1, 173 (2006).

${ }^{5}$ A. Nitzan and M. A. Ratner, Science 300, 1384 (2003).

${ }^{6}$ M. Galperin, M. A. Ratner, A. Nizan, and A. Troisi, Science 319, 1056 (2008).

${ }^{7}$ J. Hihath, C. R. Arroyo, G. Rubio-Bollinger, N. Tao, and N. Agrait, Nano Lett. 8, 1673 (2008).

${ }^{8}$ A. N. Pasupathy et al., Nano Lett. 5, 203 (2005).

${ }^{9}$ S. W. Wu, G. V. Nazin, X. Chen, X. H. Qiu, and W. Ho, Phys. Rev. Lett. 93, 236802 (2004).

${ }^{10}$ G. V. Nazin, S. W. Wu, and W. Ho, Proc. Natl. Acad. Sci. USA 102, 8832 (2005).
${ }^{11}$ X. H. Qiu, G. V. Nazin, and W. Ho, Phys. Rev. Lett. 92, 206102 (2004).

${ }^{12}$ E. A. Osorio, K. O’Neill, N. Stuhr-Hansen, O. F. Nielsen, T. Bjørnholm, and H. S. J. van der Zant, Adv. Mater. 19, 281 (2007).

${ }^{13}$ B. C. Stipe, M. A. Rezaei, and W. Ho, Science 280, 1732 (1998).

${ }^{14}$ H. Song, Y. Kim, Y. H. Jang, H. Jeong, M. A. Reed, and T. Lee, Nature (London) 462, 1039 (2009).

${ }^{15}$ J. G. Kushmerick, J. Lazorcik, C. H. Patterson, R. Shashidhar, D. S. Seferos, and G. C. Bazan, Nano Lett. 4, 639 (2004).

${ }^{16}$ L. H. Yu, Z. K. Keane, J. W. Ciszek, L. Cheng, M. P. Stewart, J. M. Tour, and D. Natelson, Phys. Rev. Lett. 93, 266802 (2004).

${ }^{17}$ A. S. Blum et al., Nat. Mater. 4, 167 (2005).

${ }^{18}$ J. Repp, P. Liljeroth, and G. Meyer, Nat. Phys. 6, 975 (2010).

${ }^{19}$ K. Moth-Poulsen and T. Bjørnholm, Nat. Nanotechnol. 4, 551 (2009).

${ }^{20}$ A. Troisi and M. A. Ratner, Small 2, 172 (2006). 
${ }^{21}$ C. Benesch, M. Cizek, J. Klimeš, I. Kondov, M. Thoss, and W. Domcke, J. Phys. Chem. C 112, 9880 (2008).

${ }^{22}$ J. Park et al., Nature (London) 417, 722 (2002).

${ }^{23}$ S. Ballmann, W. Hieringer, D. Secker, Q. Zheng, J. A. Gladys, A. Görling, and H. Weber, Chem. Phys. Chem. 11, 2256 (2010).

${ }^{24}$ F. M. Leibsle, S. S. Dhesi, S. D. Barrett, and A. W. Robinson, Surf. Sci. 317, 309 (1994).

${ }^{25}$ C. F. Hirjibehedin, C. P. Lutz, and A. J. Heinrich, Science 312, 1021 (2006).

${ }^{26}$ W. Wang, Y. Hong, X. Shi, C. Minot, M. A. Van Hove, B. Z. Tang, and N. Lin, J. Phys. Chem. Lett. 1, 2295 (2010).
${ }^{27}$ W. Wang, X. Shi, C. Lin, R. Q. Zhang, C. Minot, M. A. Van Hove, Y. Hong, B. Z. Tang, and N. Lin, Phys. Rev. Lett. 105, 126801 (2010).

${ }^{28}$ N. P. Guisinger, N. L. Yoder, and M. C. Hersam, Proc. Natl. Acad. Sci. USA 102, 8838 (2005).

${ }^{29}$ D. Porath, Y. Levi, M. Tarabiah, and O. Millo, Phys. Rev. B 56, 9829 (1997).

${ }^{30}$ S. Braig and K. Flensberg, Phys. Rev. B 68, 205324 (2003).

${ }^{31}$ A. T. Collins, E. C. Lightowlers, and P. J. Dean, Phys. Rev. 158, 833 (1967).

${ }^{32}$ J. G. Hou, B. Wang, J. L. Yang, X. R. Wang, H. Q. Wang, Q. S. Zhu, and X. D. Xiao, Phys. Rev. Lett. 86, 5321 (2001). 